\title{
Review of: "HLAncPred: A method for predicting promiscuous non-classical HLA binding sites"
}

\author{
Paulin Sonon
}

Potential competing interests: The author(s) declared that no potential competing interests exist.

\section{Study strongness}

In order to predict the promiscuous binders for the non-classical HLA alleles, Dr. Dhall et al. developed a web server HLAncPred

(https://webs.iiitd.edu.in/raghava/hlancpred), and a standalone package. Another main aim of the their study was to facilitate the scientific community, for the prediction of binders of non-classical HLA alleles (HLA-G and HLA-E), since limited attempts have been made to develop methods for predicting binders for these non-classical HLA and the scarcity of sufficient experimental data and the lack of community interest. All the models were trained and tested on experimentally validated data.

Interestingly, authors have validated their models by predicting the non-classical HLA binders in the spike protein of different variants of virus causing COVID-19 including omicron (B.1.1.529).

\section{Some points to be improved}

Line 327. ---Absence of the model training using the previous HLA-E dataset. Beside the limited HLA-E peptide in MHCflurry 2.0 and NetMHCpan 4.1 datasets, is not there any other way to also validate your models regarding HLA-E alleles?

Line 414. Discussion and Conclusion---In my point of view, it lacks the discussion. Line 464. Figure 4.---please, change figure 4 into figure 5. 